\section{Cahiers de Narratologie}

Analyse et théorie narratives

$10.2 \mid 2001$

La voix narrative

\title{
L'ironie des voix superposées dans le Gil Blas de Lesage
}

Jacques Wagner

\section{(2) OpenEdition}

1 Journals

Édition électronique

URL : http://journals.openedition.org/narratologie/10319

DOI : 10.4000/narratologie.10319

ISSN : 1765-307X

Éditeur

LIRCES

\section{Édition imprimée}

Date de publication : 1 janvier 2001

Pagination : 509-524

ISBN : 2914561032

ISSN : 0993-8516

\section{Référence électronique}

Jacques Wagner, "L'ironie des voix superposées dans le Gil Blas de Lesage », Cahiers de Narratologie

[En ligne], 10.2 | 2001, mis en ligne le 01 janvier 2001, consulté le 11 juin 2020. URL : http://

journals.openedition.org/narratologie/10319; DOI : https://doi.org/10.4000/narratologie.10319 


\title{
L'IRONIE DES VOIX SUPERPOSÉES DANS LE GIL BLAS DE LESAGE
}

\author{
Jacques WAGNER \\ Université de Clermont-Ferrand
}

«J'espérais que je passerais pour avoir de l'esprit, quoique je n'eusse que de la mémoire...Ne parlez pas si haut, interrompit en riant ma maîtresse...Dieu soit loué! puisque la mèche est découverte...changeons de style "

( $G B$, IV , 2, p. 196-197 et 11, p. 273)

Lointain est le temps où un Montaigne parvenait à unifier deux personnes distinctes au point de faire disparaître la trace même d'un lien: "En l'amitié de quoi je parle, elles (les âmes) se mêlent et confondent l'une en l'autre, d'un mélange si universel qu'elles effacent et ne retrouvent plus la couture qui les a jointes. » (Essais, I, 28, Garnier, p. 203-204) ; à cette exceptionnelle image de l'unité recomposée (pour faire allusion à la nostalgie platonicienne de l'un), s'opposerait le drame de la personne incapable de se ressembler ni de se rassembler : « Moi à cette heure, moi à cette autre sommes bien deux ». G. Poulet avait souligné cette pluralité de l'être montaignien «multiplié en une série d'apparitions et de disparitions isolées » (Études sur le temps humain, Plon, 1972, p. 49). Toutefois Montaigne maintenait la présence d'un "patron au-dedans auquel toucher nos actions, et, selon celui-ci, nous caresser tantôt, tantôt nous châtier » (Essais, III, 2, p. 225). Cette tentative de restauration empirique de l'unité par le biais de la volonté morale impliquait l'existence d'un jugement ferme et le respect de ses assertions 1 .

${ }^{1}$ Lorsque Rodrigue titube sous le coup de la nouvelle «imprévue aussi bien que mortelle ", il finit par reconnaître son erreur et par s'engager dans la seule voie acceptable : «Oui, mon esprit s'était déçu. 
Qu'en fut-il au XVIIIe ? Les récits en première personne, dits "homodiégétiques», jouent alors sur l'écart chronologique qui sépare l'acteur et le narrateur pour instiller des doses plus ou moins grandes de différences, interposant des divergences de conscience entre eux deux. Le récit manifeste trois types d'écarts : ou il explicite la coexistence de deux voix distinctes (modalité de la dissociation)2 ${ }^{2}$ ou il établit deux voix parallèles et contrastées (modalité de l'association) ${ }^{3}$, ou enfin il superpose deux voix dans la même émission verbale (modalité de la superposition), technique fréquente chez Lesage.

Notre étude sera exclusivement consacrée à cette dernière. Elle consiste à insuffler une dose suffisante d'ironie dans l'expression de la réalité vécue par le personnage pour signifier au lecteur que la représentation est soumise à une perspective inappropriée (erreur d'appréciation) ou qu'elle relève d'un jeu de masques, volontaires ou inconscients, inspiré par la tradition littéraire : la voix narrative (seconde) raconte moins une histoire qu'elle n'évalue les effets comiques et inacceptables, sur le genre romanesque, de l'esprit romanesque propre aux voix narratrices (premières).

\section{I - Pathologie de l'éloquence enthousiaste}

Dans le cas de la dissociation, deux voix séparées textuellement, interviennent, signalées par une syntaxe de l'implication conditionnelle. Le narrateur apprécie avec humour ou agacement les faits et gestes de son personnage, lui-même encore jeune : il discerne le manque d'expérience et

Je dois tout à mon père avant qu'à ma maîtresse...Je m'accuse déjà de trop de négligence ; / Courons à la vengeance ; / Et tout honteux d'avoir tant balancé, / Ne soyons plus en peine » ( Le Cid, II, 1, vers 341-348). Mme de Clèves obéira de même aux évidences de son jugement relatif à sa passion pour Nemours : «Qu'en veux-je faire...Il faut m'arracher de la présence de M. de Nemours; il faut m'en aller à la campagne...Elle demeura dans cette résolution...Il faut que je demeure dans l'état où je suis et dans les résolutions que j'ai prises de n'en sortir jamais. » Mme de La Fayette, La Princesse de Clèves (Livre de Poche, 1958), p. 148-233.

2 Modalité généralement utilisée par Crébillon dans les Egarements. - ${ }^{3}$ Modalité utilisée par Voltaire dans les Lettres philosophiques. 
se permet de l'indiquer dans le courant du récit. Gil narrateur se permet ainsi une intrusion d'auteur pour se démarquer de son personnage trop stupide à son goût: "Pour peu que j'eusse eu d'expérience, je n'aurais pas été la dupe de ses démonstrations ni de ses hyperboles; j'aurais bien connu, à ses flatteries outrées, que c'était un de ces parasites que l'on trouve dans toutes les villes. » $(G B, \mathrm{I}, 2$, Garnier, p. 9) Le narrateur suspend un instant son récit pour expliquer son attitude antérieure comme s'il s'adressait à une conscience avertie (la sienne ou celle du lecteur) ${ }^{4}$, une conscience rétive devant une telle sottise ${ }^{5}$. La voix du savoir et la voix de l'ignorance sont donc bien distinctes: l'une réécrit une version clarifiée et rectifiée d'un passé inintelligible pour le héros ou inacceptable pour le mémorialiste ; l'autre se réjouit d'un présent dont son inexpérience, sa crédulité et sa vanité faussaient la perception. Le genre des Mémoires favorise cette modalité de l'écart mais n'en exclut pas la seconde, qui superpose les deux voix.

En effet, il arrive que les deux voix se confondent. Leur rapprochement s'effectue de deux manières : à partir du personnage ou à partir du narrateur.

\section{a) Gil chez les voleurs : éloge ou flatterie ?}

Le personnage peut se mettre à parler comme le narrateur. Gil Blas, au moment même de l'action diégétique, s'efforce de résister à la tentation d'être séduit par les propos élogieux qu'on lui adresse. Devant les voleurs qui en font leur prisonnier honteux, il se croit en mesure de tirer les

${ }^{4}$ Ce schéma sera aussi celui de Meilcour dans les Égarements du coeur et de l'esprit: "J'étais trop jeune pour sentir combien ce système était absurde et pour savoir combien il était peu suivi par celles mêmes qui le soutenaient avec le plus d'ardeur. » (55) Seules les nuances y sont plus riches. Meilcour varie les supports de la double voix. Parfois, elle concerne les situations : «Je n'en savais pas assez pour nous tirer d'un état si pénible » (Seuil-Ecole des lettres, p. 92).

5 Attentif aux questions de vraisemblance, Crébillon explicite une même démarche que Lesage : "Ne connaissant pas la différence qu'il y a entre une femme vertueuse et une prude, il n'était point étonnant (spm) que je n'attendisse pas de Mme de Lursay plus de facilité qu'elle ne se disait capable d'en avoir. " (55). 
conséquences de la leçon que lui a donnée le parasite ou l'hôtelier : "J'eus le bonheur de m'attirer des compliments... Mais j'étais alors revenu des louanges et j'en pouvais entendre sans péril. » (I, 7, p. 17). Le sentiment de satisfaction semble ne pas altérer la perception de la réalité : l'opposition de la voix expérimentée ("Mais j'étais revenu... ») neutralise l'effet de perspective amorcé par la vanité («j'eus le bonheur.. »).Toutefois, cette lucidité est momentanée. Un second mouvement engage un retour contrôlé à la rhétorique de la vanité ; le narrateur insiste sur les compliments comme si le récit reprenait à son compte les émotions originelles du personnage, comme si la voix narrative en épousait les anciennes réactions intimes et quittant le registre réflexif de la distance critique, introduisait dans le texte un effet de focalisation interne: "Là-dessus, ils me louèrent tous; ils dirent que je paraissais né pour être leur échanson, que je valais cent fois mieux que mon prédécesseur. " (ibid.). Les marques linguistiques de la focalisation seraient de trois types: d'abord lexical, dans le passage du verbe hyperonyme (louer) à la liste de ses contenus spécifiques (dire que) qui indique le refus inconséquent d'une litote qui aurait été davantage conforme à la modestie de prudence revendiquée ; ensuite grammatical, dans le recours au soulignement quantitatif (tous) et à la comparaison graduée intensive (cent fois mieux); et enfin sémantique dans l'usage d'une isotopie appréciative aristocratique (la valeur de naissance et la supériorité du savoir-faire); le tout dans une atmosphère hédoniste qui rapproche plaisirs des mots et service du vin, échauffement de la tête et du corps ${ }^{6}$ : Lesage dénonce sans faiblir la fascination pour l'image superbe qui frappe l'imagination car quand elle est gagnée, le jugement recule et la vertu cède ${ }^{7}$. Le narrateur s'amuse à confronter son héros

${ }^{6}$ Crébillon établira un rapport identique entre les deux ivresses : "Abdalathif, noyé dans le vin, enivré des éloges que le mérite qu'on avait découvert à son cuisinier avait rendus plus vifs et plus nombreux, ne tarda point à s'endormir. " (Le Sopha, UGE, 1966, p. 70)

7 Un des amis de Gil, Raphaël, saura par la suite utiliser cette faiblesse humaine, plus active, d'après lui, chez les femmes; il parviendra à détourner une Lucrèce moderne de son premier époux en 
avec ses dires, le prend en flagrant délit de faiblesse vaniteuse et suggère que les compliments ont produit un effet sur son amour-propre; évitant tout recours à une démarche psychologique, il se contente d'insuffler à sa phrase une tonalité noble grâce à un lexique de style élevé par sa nature culturelle et ses allusions mythologiques: "La senora Léonarde avait l'honneur de présenter le nectar à ces dieux infernaux (les bandits du souterrain dont il est le prisonnier), ils la privèrent de ce glorieux emploi pour m'en revêtir. Ainsi nouveau Ganymède, je succédai à cette vieille Hébé » (ibid.). Les énoncés s'ouvrent progressivement à une série de substitutions hyperboliques : le nectar pour le vin, les dieux infernaux pour les voleurs, Ganymède pour l'échanson, Hébé pour Léonarde; elles traduisent l'envahissement du langage par une subjectivité que l'ivresse d'une fausse gloire (servir des voleurs de grands chemins) pousse à une exaltation burlesque. L'écart tient à l'usage d'un vocabulaire épique dans un contexte picaresque. Le narrateur rend sensible, par un jeu verbal sans contenu, la dualité des voix superposées: l'une reprend le style naturellement grandiloquent de l'éloge ; l'autre en désigne et en dénonce l'infatuation littéraire : l'expression poétique (épique) serait un agrandissement illégitime de la réalité basse dont le roman rend compte. La forme se détache du contenu comme une peau morte, ou une parure grotesque ${ }^{8}$. Le narrateur, revenu des éloges qu'il sait réduire à des flatteries illusoires, à des chants dangereux de sirène, en ridiculise la rhétorique académique : il marque une distance sarcastique à l'égard d'un style qui embellit la réalité de façon mécanique et intéressée. Il interdit à son personnage d'adhérer à des jeux de langage d'un mauvais goût assuré par des procédures de

lui parlant d'un grand-duc florentin : "Elle ne me parut pas éprise de lui, je m'aperçus néanmoins que la vanité l'empêchait de rejeter ses soupirs. Elle prenait plaisir à les entendre sans vouloir y répondre. Elle avait de la sagesse, mais elle était femme ; et je remarquais que sa vertu cédait insensiblement à l'image superbe de voir un souverain dans ses fers. » (VI, 1, p. 318-319)

8 Remarquons que Hébé, ici assignée à représenter la vieille Leonarde, était la déesse de la jeunesse. 
soulignement (hyperboles) et de déplacement (connotations nobles dans un contexte bas).

\section{b) Gil chez les comédiens : beauté divine ou erreur humaine ?}

La hantise du beau et du grand accapare les personnages du $G B$ qui oublient qu'ils ne sont plus que les personnages d'une « histoire » et non d'un « roman»?.

Arsénie, la comédienne, en est l'exemple-type qui joue encore l'ancien rôle de la dépense magnifique et ridicule ${ }^{10}$; quand Gil entre à son service, il découvre ses appartements avec une stupéfaction où se mêlent l'appétit de luxe d'un jeune homme pauvre et le bon goût d'un narrateur ironique : "Quel luxe! quelle magnificence! Je me crus chez une vice-reine, ou, pour mieux dire, je m'imaginai voir toutes les richesses $d u$ monde amassées dans un même lieu. Il est vrai qu'il y en avait de plusieurs nations, et qu'on pouvait définir cet appartement le temple d'une déesse où chaque voyageur apportait pour offrande quelque rareté de son pays. J'aperçus la divinité assise sur un gros carreau de satin, je la trouvai charmante et grasse de la fumée des sacrifices. " (GB, III, 10, p. 179-180). Le texte fait «mention » des attitudes admiratives liées topologiquement à la description : la syntaxe exclamative, la langue hyperbolique, les comparaisons flatteuses, la thématique des richesses accumulées sont systématiquement affaiblies par des signaux intertextuellement lisibles : «magnificence », mot noble qui introduit la description de la cour dans la Princesse de Clèves, est précédé de

${ }^{9}$ L'opposition est à la mode dès la fin du XVII siècle. Voltaire l'utilise dans un parallèle qui oppose Locke à la tradition idéaliste : "Tant de raisonneurs ayant fait le roman de l'âme, un sage est venu qui en a fait modestement l'histoire. "(Lettres philosophiques, 13, GF, p. 83)

10 Fontenelle oppose l'épargne naturelle à la dépense humaine : « Nous autres, nous sommes sujets à renverser souvent tout cela dans nos idées. Nous mettons l'épargne dans le dessein qu'a eu la nature et la magnificence dans l'exécution. Nous lui donnons un petit dessein qu'elle exécute avec dix fois plus de dépense qu'il ne faudrait ; cela est tout à fait ridicule. » (ed. Gérard, 1973, p. 36) 
" luxe », terme économique" étranger au monde romanesque produit une rencontre lexicale où s'entrechoquent deux univers hétérogènes; le narrateur aplanit la différence de niveau, efface la hiérarchie des styles et fait de Gil un esprit naïf qui conserve les formes stéréotypées de l'admiration sans maîtriser les connotations des synonymes, comme s'il mélangeait, grâce à une inadvertance feinte du narrateur, les torchons et les serviettes, l'aristocratie et la bourgeoisie, le style romanesque et la philosophie économique. Les failles du système rhétorique sont agrandies par la posture de l'énonciateur : entre le réel et le langage, ou il place des mots indiquant son illusion : « je me crus, je m'imaginai »; ou il soumet sa première expression ("vice-reine ») à une traduction qui lui enlève son idéalité abstraite conforme à l'esthétique classique de la généralité qualitative et la ramène à une réalité matérielle dénombrable: "toutes les richesses du monde amassées » et inversement ennoblit l'appartement en temple de déesse antique ; le produit de ces métamorphoses se synthétise dans l'expression finale qui élimine toute distance explicite en substituant à l'illusion une perception immédiate ( «j'aperçus ») mais répond à la double isotopie, mise en place auparavant, de la sphère qualitative de l'idéalité («la divinité ») et de la sphère quantitative de la matérialité («un gros carreau de satin ») et à un jeu du contraste hétérogène (« charmante et grasse de la fumée des sacrifices »).

Lesage est un spécialiste du recours à la voix de l'autre; il a montré son Gil prenant le ton des voleurs jusqu'au ridicule ; puis la voix d'Ârsénie jusqu'au burlesque ${ }^{12}$. Ces imitations

11 Voir André MORIZE : L'apologie du luxe au XVIIIe et le Mondain de Voltaire, Didier, 1909 ; voir surtout pour la période de Lesage, B. Mandeville : La ruche mécontente ou la conversion des coquins à l'honnêteté (1705) dont le titre changera en la Fable des abeilles (1714); le livre eut assez de succès pour que Lesage en connût au moins le titre ; à défaut, il aurait pu s'inspirer, comme ailleurs, de son auteur préféré, La fontaine : "Je ne sais d'homme nécessaire/ Que celui dont le luxe épand beaucoup de bien. »(Fables, VIII, 19)

12

Lesage se montre aussi caricatural probablement par rancoeur à $l^{2}$ égard des comédiens qui l'avaient maltraité comme auteur 
dépeignent son attirance autant que sa vanité et laissent résonner la voix silencieuse d'un commentaire amusé.

\section{II - Un jeu de masques : éloquence tragique ou comédie bouffonne?}

Dans d'autres cas, elle ne peint que l'habileté de celui qui emprunte la voix de l'autre.

Raphaël, cherchant à convaincre sa mère Lucinde de quitter Alger avec lui et sa jeune sœur alors qu'elle choisit contre toute attente d'y rester par amour, recourt au style grandiloquent de la tragédie: "Que vois-je, lui dis-je, d'où vient que vous m'offrez un visage épouvanté ? ... Qu'entends-je?, m'écriai-je avec douleur; ah! dites-moi plutôt que c'est l'amour qui vous en détache. Quel changement, ô ciel! ... Quel projet! interrompis-je avec horreur...Ah! ma mère que me faites-vous envisager? Vous avez résolu votre perte...Ah! malheureuse Lucinde, si rien n'est capable de vous retenir, abandonnez-vous du moins toute seule à la fureur qui vous possède; n'entraînez point une jeune innocente dans le précipice où vous courez vous jeter. " $(\mathrm{V}$, I, p. 314-315) ; ce style inattendu, dans la bouche de Raphaël davantage porté à l'humour intertextuel ${ }^{13}$, est une imitation volontaire d'un style correspondant au rôle tragique du sacrifice par amour que revêt soudain Lucinde déjà trois fois veuve mais farouchement décidée à dénoncer son fils auprès du pacha. Raphaël donne la clé de la scène : "Je ne doutais pas un moment que Lucinde ne fût femme à (le) faire). J'avais eu le temps d'étudier la dame et je m'étais aperçu qu'à force de jouer des rôles sanguinaires dans les

dramatique; mais le système ironique de la double voix reste conforme à l'esprit de son narrateur.

13 Racontant ses mésaventures maritimes, il adopte l'attitude plaisante des romanciers «comiques » du XVIIe usant de la prétérition à l'égard des grandiloquences romanesques: «A peine fûmes-nous hors du golfe d'Alicante qu'il survint une bourrasque effroyable. J'aurais, dans cet endroit de mon récit, une occasion de vous faire une belle description de tempête, de peindre l'air tout en feu, de faire gronder la foudre, siffler les vents, soulever les flots, etc...; mais laissant à part toutes ces fleurs de rhétorique, je vous dirai que l'orage fut violent. » $(\mathrm{V}, 1, \mathrm{p} .295)$ 
tragédies, elle s'était familiarisée avec le crime. Elle m'aurait bien fait brûler tout vif; et je ne crois pas qu'elle eût été plus sensible à ma mort qu'à la catastrophe d'une pièce de théâtre. "(ibid.) Dans le même ensemble textuel s'insinue l'équivalent d'une connotation générique (style du théâtre tragique) et d'une connotation parodique (style utilisé comme un rôle) : la voix du romancier ironique subsiste sous la couche pathétique et se démasque en révélant l'inclination tragique de la mère comédienne ; la double voix relève alors de la rhétorique, d'une intention persuasive et d'une volonté délibérée d'efficacité à l'intérieur d'une comédie " littéraire » où les rôles sont des masques et les mots des jeux de langage qui construisent des images flatteuses pour échauffer l'esprit et le séduire ${ }^{14}$. Elle est alors citationnelle et pragmatique.

\section{III - La bêtise romanesque : les châteaux en Espagne du roman}

Parfois elle est seulement culturelle : hérité inconsciemment par éducation, l'esprit romanesque façonne la personnalité de Gil et lui impose une manière d'imaginer le monde. Doté d'une riche culture scolaire, il aime à se considérer comme un « illustre » digne de jouir des privilèges qu'il associe au statut noble.

Aussi lorsque Raphaël et Camille l'invitent dans leur supposé château, ne résiste-t-il pas à la tentation; ses deux partenaires l'enveloppent des signes matériels et verbaux de la beauté, du luxe et de la grandeur. Il y adhère sans recul ni méfiance malgré un premier réflexe critique: «Je me ressouvins du parasite de Penaflor et j'allais soupçonner la dame d'être une franche aventurière ; mais ce qu'elle ajouta m'en fit juger plus avantageusement. » (I, 16, p. 58). Il s'enfonce donc dans sa

14 Gil BLAS, aussi, a eu recours aux mots du théâtre pour séduire une femme mais sur un mode plaisant. Avant de se rendre à ce qu'il croit être un rendez-vous amoureux avec Aurore, une dame de condition, il se prépare à adopter un rôle de "galant poli, tendre et respectueux sans pourtant être timide »: "Je me représentais qu'en peu de temps j'aurais le plaisir de me voir aux pieds de cette aimable dame et de lui dire mille choses passionnées. Je rappelai même dans ma mémoire tous les endroits de nos pièces de théâtre dont je pouvais me servir dans notre tête-à-tête et me faire honneur. » (IV, 1, p. 196) 
fiction sans se rendre compte qu'il multiplie pour le lecteur les signes de sa bêtise vaniteuse : il est flatté d'être considéré en seigneur d'importance que l'on recherche et que l'on respecte, il note la qualité de la maison où il est invité, il dénombre les bougies qui éclairent l'appartement, il apprécie la quantité de domestiques qui l'accueillent, mais la description est affectée d'un coefficient restrictif d'insatisfaction discrètement suggérée : la maison est « assez grande », l'appartement pas «malpropre » (=non sans élégance), le compte des bougies n'est pas établi sans hésitation (vingt ou trente), les domestiques sont plusieurs et non pas nombreux ; cette réalité qui n'a rien pour exalter un esprit romanesque, n'ouvre pas les yeux de Gil mais ceux du lecteur; enfermé dans son désir, Gil perd la conscience de la réalité ; c'est pourquoi, Lesage le montre rassuré et comblé dans son appétit de grandeur par les mots qui constituent l'essentiel de sa réalité : le faux seigneur qui le reçoit « rend grâce au ciel » de leur " heureuse rencontre ", et le serrant étroitement dans ses bras ajoute avec emphase : "Quelle joie j'ai de voir ici le seigneur Gil Blas de Santillane! Il n'était pas besoin que ma cousine la marquise nous recommandât de vous régaler; elle n'avait seulement qu'à nous mander que vous deviez passer à Valladolid : cela suffisait » (I, 16, p. 59). Le repas mêle ivresse des mots et gaîté du vin. Enfin le château se réduit à une "description magnifique » complétée par des promesses alléchantes: il «me parla des plaisirs qu'il prétendait m'y donner. Tantôt, disait-il, nous prendrons le divertissement de la chasse, tantôt celui de la pêche; et si vous aimez la promenade, nous avons des bois et des jardins délicieux. D'ailleurs nous aurons bonne compagnie... J'acceptai la proposition. » (ibid. p. 60). Raphaël ne fait que dévider le contenu de l'expression : "la vie de château »15 dont le roturier rêve comme le petit bourgeois de Barthes se fabriquait, à la vue des rois en croisière, « une certaine idéalité

15 Littré déroule ainsi le contenu de l'expression : «à la campagne dans une maison riche et amie où l'on trouve tous les plaisirs du lieu (pêche, chasse, bonne table...), ce qui correspond aux affirmations de Raphaël. 
de la vie quotidienne »16. L'être romanesque de Gil est rapidement confronté à la dure loi du réel : « Je fus alors au fait »; au lieu d'être dans une grande maison pleine de domestiques, il est dans un "hôtel garni » et au lieu de posséder un vrai bijou $^{17}$ et un habit de cavalier, il est privé de tout. Le lecteur percevait sous le récit de la fascination roturière la petite voix du mémorialiste averti du danger des images flatteuses : au moment où les personnages procèdent au troc des bagues, le narrateur offre, avec la hâte et la sortie brusque de Camille, des indices clairs de duplicité que Gil ne décrypte pas tant il est aveuglé par «les douces œillades»de la dame et ses illusions amoureuses : "Puisque le rubis vous plaît, je veux faire un troc avec vous. Aussitôt elle prit ma bague et mit la sienne au petit doigt. Après ce troc qui me parut une manière galante de faire un présent, Camille me serra la main et me regarda d'un air tendre; puis tout à coup, rompant l'entretien, elle me donna le bonsoir et se retira toute confuse, comme si elle eût honte de me faire trop connaître ses sentiments. "Sa vanité l'a rendu sensible à des fictions : "Plein de cette idée flatteuse et de l'état brillant de mes affaires, je m'enfermai dans la chambre où ...je $m$ 'abandonnai aux réflexions agréables que ma valise et mon rubis m'inspirèrent. » (p. 61) ${ }^{18}$. L'insistance du narrateur sur la confusion chez Gil entre sa représentation du monde et la réalité installe la seconde voix dans le récit, une seconde voix qui avertit en vain l'entendement des risques de méprise interprétative : "Il me semblait quelquefois que Camille qui trinquait avec nous, me lançait des regards qui signifiait quelque chose. Je crus même remarquer qu'elle prenait son

16

Dans les Mythologies. (1957) de Barthes, l'article intitulé « La croisière du sang bleu » détaillait le rêve de belle vie fait par les simples mortels voués au prosaïsme démocratique; (Seuil-Points, 1970, p. 35).

17 Parallèlement à la promesse de mener une vie de château, Camille lui offre une bague venue des îles Philippines estimée trois cents pistoles, ce qui renforce la connotation romanesque par la référence aux îles lointaines du Pacifique.

18 Comment ne pas penser aux fables que La Fontaine consacre aux erreurs de l'imagination anticipatrice chez Perrette (VII, 10) ou chez les deux compagnons (V, 20). 
temps pour cela, comme si elle eût craint que son frère ne s'en aperçut. Il n'en fallut pas davantage pour me persuader que la dame en tenait et me flattait de profiter de cette découverte. » (p. 60). Les équivoques d'interprétation sont ironiquement justifiées par le narrateur qui souligne sa prime incompétence de déchiffreur: "Quoique galant des plus novices ${ }^{19}$, je sentis tout ce que cette retraite précipitée avait d'obligeant pour moi et je jugeai que je ne passerais point mal le temps à la campagne »(p. 61). Pure construction de l'esprit, le bonheur matériel et sentimental est présenté par les seuls biais de l'imagination et de la promesse : "Les bontés de cette généreuse dame se présentaient à mon esprit avec tous leurs charmes, et je goûtais aussi par avance les divertissements que don Raphaël me préparaient dans son château. »(p. 61). Lesage dénonce les emballements romanesques du désir: "Grâce au ciel, disai-je, si j'ai été malheureux, je ne le suis plus...me voilà pour longtemps en fonds.. j'enflammerai mille femmes à Madrid puisque j'ai plus si facilement à Camille. » (p. 61). L'aspect littéraire du comportement intellectuel de Gil inspire au narrateur une indexation moqueuse $\mathrm{e}^{20}$ : «Cependant, parmi tant d'images de plaisir, le sommeil ne laissa pas de venir répandre sur moi ses pavots. " L'intrusion, incongrue pour le lecteur, de ce stéréotype poético-épique mime verbalement le délire compensatoire de Gil tout en précédant l'ouverture à une conscience dégrisée du réel : "Je compris que mon valet, ayant une entière connaissance de mes affaires, m'avait vendu à ces fourbes» (p. 62). Toutefois la conscience

${ }^{19}$ Ce mouvement de phrase fait penser au Sopha de Crébillon : «Malgré le peu d'expérience de Philéas, sa tendresse qui le rendait attentif à tous les mouvements de Zéïnis, les lui laissait assez remarquer pour qu'il ne pût pas douter qu'elle le voyait avec plaisir. " (UGE, 1966, p. 290). La différence tient au scepticisme de Lesage qui ne voit dans l'amour que fausseté du jugement et erreur d'appréciation (voir la note sur la conception de l'amour chez Mergelina).

20

La Fontaine que Lesage semble connaître par coeur s'était déjà amusé de la formule : "Il (le renard) choisit une nuit libérale en pavots ; / Chacun était plongé dans un profond repos ; / Le maître du logis, les valets, le chien même, / Poules, poulets, chapons tout dormait. » (Fables, XI, 3) 
désabusée ne trouve pas immédiatement son équivalent verbal. Le personnage continue à s'exprimer, sous la pression de son héritage culturel ${ }^{21}$, à la manière d'un être de fiction, victime de la fatalité : "Au lieu de n'imputer qu'à moi ce triste incident et de songer qu'il ne me serait point arriver si je n'eusse pas eu l'indiscrétion de m'ouvrir à Majuelo (l'hôtelier) sans nécessité, je m'en pris à la fortune innocente et maudis cent fois mon étoile ». (ibid., p. 62). Un caractère littéraire 22 persiste dans la voix narrative première.

Ainsi donc, dans le Gil acteur s'obstine une voix romanesque dont se détache le narrateur second plus proche des sévérités jansénistes que des rêveries hédonistes et de leurs pendants linguistiques. Les beautés littéraires sont devenues des beautés de village, pour reprendre les termes d'une critique pascalienne ${ }^{23}$.

21 Il est constitué essentiellement d'éléments classiques comme le signale la définition que Mergelina donne de l'amour et les conséquences subjectives qu'elle implique: "L'amour est un dérèglement d'esprit qui nous entraîne vers un objet et nous y attache malgré nous : c'est une maladie qui nous vient comme la rage aux animaux. Cessez donc de me représenter que Diego n'est pas digne de ma tendresse ; il suffit que je l'aime pour trouver en lui mille belles qualités qui ne frappent point votre vue et qu'il ne possède peut-être pas. (II, 7, p. 112)

22 Gil est réputé pour sa culture : «puisque Gil Blas est un garçon qui a déjà de la littérature, pour achever de le rendre savant, je lui laisse une bibliothèque, tous mes livres et mes manuscrits, sans aucune exception ", écrit Sédillo dans son testament (II, 2, p. 80).

${ }^{23}$ Dans les Lettres philosophiques, 25 (GF, 1964, p. 185), Voltaire, au contraire de Lesage, rejettera le refus janséniste de " certains termes bizarres, siècle d'or, merveilles de nos jours, fatals, etc " et les moqueries de Pascal : " Et on appelle ce jargon beauté poétique. Mais qui imaginera une femme sur ce modèle-là, qui consiste à dire de petites choses avec de grands mots verra une jolie demoiselle toute pleine de miroirs et de chaînes dont il rira parce qu'on sait mieux en quoi consiste l'agrément d'une femme que l'agrément des vers mais ceux qui ne s'y connaîtraient pas l'admireraient en cet équipage et il y a bien des villages où on la prendrait pour la reine et c'est pourquoi non appelons les sonnets faits sur ce modèle-là les reines de villages. " (Pensées, 586, Papiers non classés, Seuil-Lafuma, 1963, p. 583) 


\section{Conclusion}

Lesage en s'en prenant aux tendances romanesques du personnage redresse une orientation subjective qui confond représentation et perspective : "Je jugeais de ses sentiments par les miens », avoue Diégo déçu par l'accueil que lui fit un jour son oncle, « je n'eus pas l'esprit de m'apercevoir qu'il ne cherchait qu'à m'éloigner de lui » (II, 7, p. 106-107); le texte lesagien est certes structuré par « un champ de tensions » ${ }^{24}$, mais il est très fortement vectorisé : la narration est aussi une occasion pour "se désabuser » des opinions reçues grâce aux preuves qui les contredisent (III, 12, p. 189) et pour devenir un «juste appréciateur» (ibid.): parce qu'il ne se sent " aucune disposition à s'en laisser éblouir », Gil a acquis la capacité de concevoir l'envers du récit, de confier la narration à une autre voix qui relativise l'énonciation première : «Nous fûmes servis par des domestiques du logis qui pendant le repas tandis que nous affections une gravité imposante, riaient peut-être en eux-mêmes du respect de commande qu'ils avaient pour nous. » (XI, 5, p. 282). Pour le formuler à la manière de Lesage, "le préjugé (ne) l'emporte (plus) sur l'expérience » (II, 3, p. 83); pour le formuler à la manière de La Fontaine, l'esprit ne bat plus la campagne, le narrateur ne fait plus de châteaux en Espagne ${ }^{25}$. La voix seconde sait à la suite du fabuliste que "chacun songe en veillant, qu'il n'est rien de plus doux » car " une flatteuse erreur emporte alors nos âmes; tout le bien du monde est à nous; tous les honneurs, toutes les femmes " mais que «quelque accident fait-il que je rentre en moi-même, / Je suis gros Jean comme devant » (ibid.). Pour éviter les déconvenues dues aux images de la tradition poético-romanesque, trop prometteuses pour être honnêtes, Lesage mêle dans son personnage-narrateur une première personne et une troisième personne (JE-IL) qui produit le nom textuel de Gil Blas, celle qui vit les illusions sans les percevoir (Il croyait) et celle qui les perçoit sans les

24 J'emprunte l'expression à Béda Allemann : « De l'ironie en tant que principe littéraire » (Poétique 36-1978).

${ }^{25}$ Fable VII, 10, vers 30-31. 
vivre (je comprends) ${ }^{26}$; la fusion des deux instances permet de dénoncer les confusions de la conscience diégétique (prise dans son histoire et ses crédulités).

L'intimité existentielle du narrateur homodiégétique est constamment brisée soit par la suite des événements vécus qui se chargent de mettre le héros «au fait » en l'obligeant à constater ses erreurs de jugement et d'appréciation soit par sa position extradiégétique qui le distingue de sa nature première et transforme le texte romanesque en citations parodiques : la pratique littéraire de Lesage ${ }^{27}$ fait bien du roman et du romanesque une "mention » ironique (Sperber et Wilson, Poétique 36-1978) en dissociant le mémorialiste de son héros, en renonçant à l'aliénation de l'adhésion ${ }^{28}$ dogmatique ${ }^{29}$, enfin en préférant l'esprit à la lettre ${ }^{30}$. Avec Lesage et ses

${ }^{26}$ V. JANKÉLÉVITCH, « L'ironie ne veut pas être crue, elle veut être comprise " (L'ironie, Flammarion - Champ, 1979, p. 60).

27 A suivre $\mathrm{Ph}$. HAMON, on serait tenté de penser que l'oeuvre de Lesage n'est qu'un exemple de "la littérature même » en ce qu'elle serait fondamentalement liée à l'ironie (L'ironie littéraire, Hachette, 1996, p. 41).

28 Pour O DUCROT, la parole ironique tient à la différence de rapport à l'énoncé, non pas une adhésion, une prise en charge par le locuteur mais une attribution du point de vue manifesté dans les paroles à un autre personnage (Le dire et le dit, Minuit, 1984, p. 211).

29 Je prends ce mot à J. Decottignies : « Entre voir et savoir, entre posséder et connaître, l'ironie dénonce l'écart que s'obstine à ignorer notre dogmatisme invétéré. " (Écritures ironiques, PU Lille, 1988, p. 12)

30 V. JANKÉLÉVITCH, «Faire le jeu du tricheur, c'est prendre la lettre pour de l'argent comptant et c'est être la dupe inconsciente et docile d'une fraude ; faire le jeu de l'ironie, qui est le jeu impersonnel de la vérité, c'est au contraire dire non à la lettre pour en toute lucidité, dire oui à l'esprit... écrire pour être mécompris mais finalement se faire mécomprendre pour convertir plus efficacement son prochain à ce qu'on croît être la vérité : voilà l'invisible visibilité, la transparente opacité du masque ironique. »(L'ironie, Flammarion-Champ, 1979, p. 62) 
contemporains, le roman abandonne l'esprit de sérieux ${ }^{31}$ et de vanité ${ }^{32}$ pour entrer dans l'ère de l'incrédulité rieuse et de l'humilité gaie, à la suite de Fontenelle dont il semble avoir tiré une leçon dans l'ordre littéraire : "La même inclination qui fait qu'on veut avoir la place la plus honorable dans une cérémonie fait qu'un philosophe, dans un système, se met au centre du monde s'il peut. Il est bien aise que tout soit fait pour lui ; il suppose peut-être sans s'en apercevoir ce principe qui le flatte et son cœur ne laisse pas de s'intéresser à une affaire de pure spéculation. »(p.37). Lesage détecta cet intérêt du cœur (vaniteux) dans une affaire de pure narration : il mit en scène des personnages parlant «la bouche pleine »33 de grands mots. La double voix répond donc aux exigences d'une épistémologie de l'expression « naturelle ${ }^{34}$ contestant l'institution ${ }^{35}$ romanesque. Le roman, sur le modèle de la comédie, ne se voulut-il pas, selon Crébillon, un «tableau de la vie humaine » qui en censurât " les vices et les ridicules », cad essentiellement la vanité et l'imagination?

31 La Marquise des Entretiens sur la pluralité des mondes réclame des certitudes car, reconnaît-elle, «j'ai besoin de croire » (ed. cit., p. 67).

32 FONTENELLE a annoncé cette nouveauté dans l'ordre culturel, dès ses Entretiens sur la pluralité des mondes : félicitant Copernic de ses découvertes, il le remercie "d'avoir rabattu la vanité des hommes qui s'étaient mis à la plus belle place de l'univers » (ed. Gérard, 1973, p. 37).

33 En 1975, Judith SCHLANGER avait publié Penser la bouche pleine (La Haye, Mouton) ; Lesage aurait été heureux de lire son essai sur Le Comique des idées (Gallimard, 1977) et en aurait apprécié La mémoire des æuvres (Nathan, 1992).

34

Le mot se trouve partout dans Marivaux mais aussi dans la préface des Illustres françaises (Challes, 1715).

35

Sur ce mot, voir, après BOURDIEU, VIALA A. (Les institutions de la vie littéraire en France au XVIIe, Lille, 1985; ou Littératures classiques 34-1998, p. 121) et M. Fumaroli (Trois institutions littéraires, Gallimard, 1994). 